\section{Dr. R. S. Thanabalasundaram}

Dr. R.S. Thanabalasundaram, a much loved, respected and admired physician and clinical teacher died after a brief illness in Colombo on the 15 November 2007, aged 85 years. He was an icon among physicians, and practiced medicine for over half a century with great devotion and dedication. Despite his illness, his interest in clinical medicine never wavered. He remained alert to the last, and his daughter lovingly recalls how his eyes shone when Dr. H. H. R. Samarasinghe who treated him during his last illness discussed an interesting clinical problem with him.

He was born to traditional Hindu parents in Kokuvil, Jaffna where he had his primary education. He thereafter entered Royal College, Colombo. He had a brilliant academic record at Royal College where he won many prizes and awards including the coveted "Turner Prize”, awarded for outstanding academic performance. He entered the Colombo Medical College where he continued his remarkable career.

As a medical student he lived at the Brodie hostel during which period my late father Mr. A. D. H. Samaranayake, Mr. Sam Wijesinghe, Dr. Charlie Munasinghe, Dr. Navaratnam and Dr. H. M. P. Perera were his contemporaries. My father and Mr. Sam Wijesinghe recall "Thanaballs" (as they affectionately called him) as a brilliant and diligent student.

He breezed through medical school obtaining first class honours in all examinations including the final MBBS, where he obtained distinctions in medicine, surgery and obstetrics and gynaecology. Soon after, he was successful in the MD (Cey.) examination and obtained the MRCP having won a government scholarship to the United Kingdom.

On his return he was appointed Physician of his native place Jaffna, and then as Consultant Physician to the General Hospital, Colombo. It was during this period that his popularity as an eminent and caring physician reached tremendous heights, and his ability as a clinical teacher made medical students throng his ward and its corridors during his ward classes.
I had the good fortune of doing my first clinical appointment as a third year medical student under his guidance and it was from this beloved physician that I learnt the ABC of clinical and bedside medicine. He impressed upon us the value of good history taking and complete clinical examination. Dr. Thanabalasundaram was one of the first clinicians to perform peritoneal dialysis on victims of snake bite who developed kidney failure. During such dialyses, two medical students of our group had to take turns spending the entire night in the ward, maintaining input-output charts, changing tubes and drips, etc. and return to work the next morning!

He treated all patients alike irrespective of their status. The attention and time each patient received during the ward round depended entirely on the severity of the illness or how puzzling the clinical problem was. Dr. Thanabalasundaram was an astute teacher. His definition of bronchial breathing, which I learnt from him in 1971, is better than in any textbook. It is the clinical information that I gathered from teachers like him that I have attempted to convey to medical students who I have taught at the Colombo North Teaching Hospital, in Ragama, and Sri Jayewardenepura General Hospital. He was held in high esteem by the students of the North Colombo Medical College who considered him their "father of medicine".

He was overjoyed that his grandson was selected to do medicine in the United States, and I had the pleasure of showing him clinical problems at the Sri Jayewardenepura General Hospital. When I visited him in hospital during his last illness I recalled many events of the past with nostalgia. Despite his illness, he enjoyed reminiscing.

He faced death fearlessly, as he was a man who had lived his life to the fullest. He fulfilled all his family obligations and devoted the prime of his life and his sunset years to the service of the sick and needy. Dear Sir, long after you are gone, you will live on in the memory of all your students and doctors who had the privilege of training under you. We will remember you with much love and gratitude for your commitment to clinical teaching, caring for the sick, your warmth, simplicity and humane qualities and above all, your devotion to the cause of medicine.

Anula Wijesundere 